\title{
Problems of Amplifier Klystron Advancing into the Terahertz Band
}

\author{
A.D. Grigoriev ${ }^{1,2}$ \\ ${ }^{1}$ Saint Petersburg State Electrotechnical University “LETI”, St. Petersburg, Russia, adgrigoriev@ mail.ru, \\ 2"Svetlana-Electronpribor" JSC
}

Electromagnetic oscillations and waves of the terahertz band have some important preferences compared with their neighbors. But lack of power coherent sources impedes wide application of these waves in radar and telecommunication systems.

Amplifier klystrons due to their high efficiency and relatively small dimensions are considered as one of the most perspective power sources of submillimeter and terahertz radiation. For example, the CPI Inc. (Canada) produces a number of Extented Interaction Klystrons (EIK) with working frequencies from 94 to $280 \mathrm{GHz}$ and output pulse power from 3 to $0.03 \mathrm{~kW}$ [1]. In Russia, the "Svetlana" JSC produces the 8-mm wavelength amplifier klystron with output power 6 $\mathrm{kW}$.

In spite of these advances, design and manufacturing of terahertz amplifier klystrons encounter a number of serious problems, namely:

- Very high current density of the electron beam;

- High cathode current density;

- Low cavities impedance;

- Small gap length and consequently little threshold voltage.

- Small collector area and dissipated heat.

To overcome these problems some techniques were proposed [2]. Among them are using of extended interaction (multigap) cavities, sheet electron beams and cathodes with high current density. But to obtain desirable effect the designer have to choose thoroughly the main device parameters starting from project input data.

The main input data for amplifier klystron design are its working frequency $f_{0}$, output power $P_{\text {out }}$, bandwidth $\Delta f$ and amplification factor $G$. Basing on these data, the designer have to estimate device efficiency $\eta$, which can change from 10 to $80 \%$. The more is working frequency and bandwidth, the less is efficiency.

When efficiency is estimated, one can calculate electron beam current $I_{0}=P_{\text {out }} /\left(\eta U_{0}\right)$, where $U_{0}$ - accelerating voltage. Increasing of the voltage leads to decreasing beam current density, increasing gap length and optimal value of the cavity $R / Q$ ratio. As a rule, such values cannot be achieved in a single gap cavity, so multigap cavities have to be used. Besides, one has to use complicated power sources.

Decreasing accelerating voltage, vice verse, decrease demanding $R / Q$ ratio, but increases beam current. Large magnetic field is needed to transfer such beam throw the interaction region. And cathodes with high current density have to be used.

The efficient way to overcome these difficulties is using a sheet electron beam. With a given beam current a sheet beam with cross-section $w \times d$ has current density approximately $w / d$ times less than a pencil beam with diameter $d$. But, cavities designed for interaction with a sheet beam have $R / Q$ ratio much less than cavities for a pencil beam.

The mentioned considerations were taken into account in the project of $95 \mathrm{Ghz}$ high power klystron proposed by the "Svetlana-Electronpribor" JSC in collaboration with LETI. The pulsed output power of the device is no less than $1 \mathrm{~kW}$, relative bandwidth $\geq 1 \%$ and amplification factor $\geq 45 \mathrm{~dB}$. After examining parameters of the existing devices, efficiency of the klystron was set at $10 \%$ level. It is rather low value, but taking in account possible manufacturing difficulties, this value seems reasonable.

The accelerating voltage was set to $10 \mathrm{kV}$, which is rather low compared with, for example, CPI EIKs with $U_{0}=20 \ldots 25 \mathrm{kV}$. Chosen value helps us to design klystron cavities and obtain demanded bandwidth. The beam current in our case have to be $1 \mathrm{~A}$. A pencil beam with such current has current density more than $5 \mathrm{Ka} / \mathrm{cm}^{2}$, which is unreal, so we chose sheet current with $1 \times 0.1 \mathrm{~mm}^{2}$ cross-section.

Three-gaps cavities composed from a section of wide comb slow wave system were chosen as an input and idle resonators and four-gaps cavity of the same type - as an output resonator. The working mode of the all cavities is $2 \pi$ because this mode well separated from the neighboring ones and has positive electron load, preventing self-oscillations.

To find optimal cavities configuration we used computer simulation code RFS [3], based on vector finite element method. Calculated cavity parameters are given in the table, where eigenfrequency $f_{0}, Q$ factor, $R / Q$ ratio, interaction factor $M$ and relative electron load $\mathrm{Ge} / \mathrm{G}_{0}$ are listed.

\begin{tabular}{|c|c|c|c|c|c|}
\hline $\begin{array}{c}\text { Cavity } \\
\text { type }\end{array}$ & $f_{0}, \mathrm{GHz}$ & $Q_{0}$ & $\frac{R / Q,}{\mathrm{Ohm}}$ & $M$ & $G_{e r}$ \\
\hline $\begin{array}{c}\text { Input \& } \\
\text { idle }\end{array}$ & 94.3667 & 1120 & 83.1 & 0.654 & 0.3 \\
\hline Output & 95.0617 & 1310 & 112 & 0.65 & 0.2 \\
\hline
\end{tabular}

Fig. 1 shows the computer cavity models with main dimensions. Input and output cavities are coupled with waveguides by slots in their sidewall. Ceramic rods are used for cavity tuning (not shown on Fig. 1)

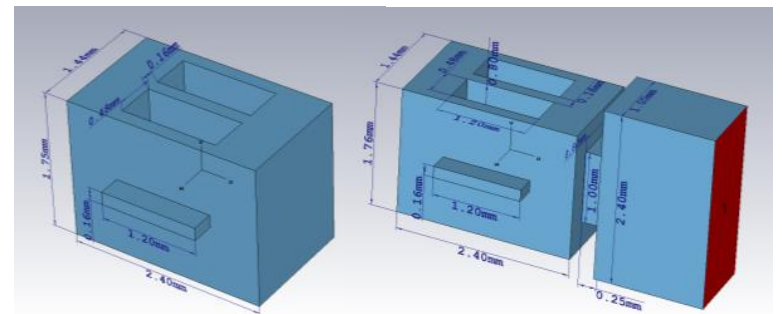

Fig. 1. Computer models of the input cavity (left) and output cavity with waveguide (right) 
Input and output cavities frequency charts are shown on Fig. 2 for beam current $0.8 \mathrm{~A}$ and accelerating voltage $10 \mathrm{kV}$. As can be seen, the output cavity coupling slot provides bandwidth about $1 \mathrm{GHz}$, i. e. almost $1 \%$.
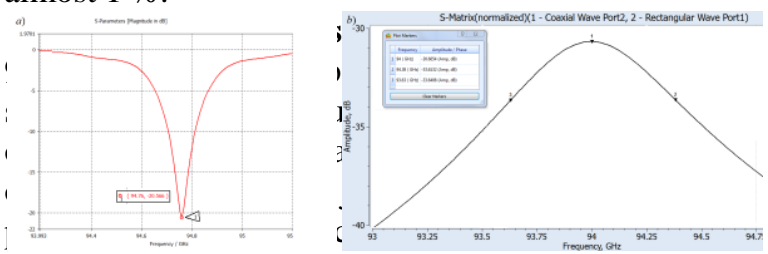

Fig. 2. Input (a) and output (b) cavities frequency charts.

The model of the klystron interaction region is shown on Fig. 3. The model contains 6 cavities, their eigen frequencies were tuned according to the skirtron scheme, where eigen frequencies of all intermediate (idle) cavities are higher than $f_{0}$. Input cavity eigen frequency is lower than $f_{0}$, and output cavity frequency is equal to $f_{0}$. Electron gun, collector and magnetic focusing system are not shown.

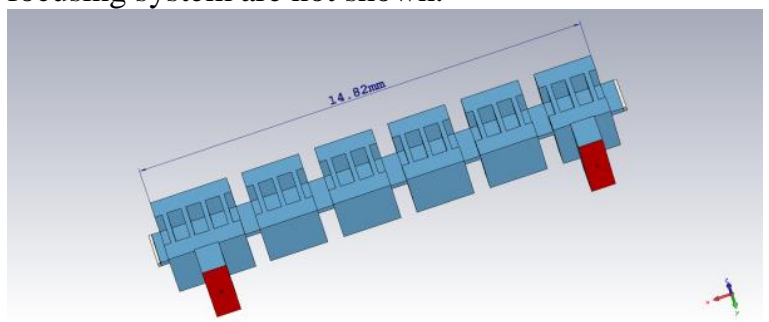

Fig. 3. Model of the klystron interaction region.

Field-particle interaction was modeled by Particle-in-Cell (PIC) method. Total number of particles in the interaction region was 26500 , simulation time 15 ns, which was sufficient to reach stationary regime. Fig. 4. Shows calculated amplitude chart of the klystron at the central frequency. Klystron amplification factor changes from $60 \mathrm{~dB}$ for small signal regime to nearly $50 \mathrm{~dB}$ for the saturation point. These values are greater than targeted one.

Output power as a function of frequency at the input power level $8 \mathrm{~mW}$ is depicted on Fig. 5. The -3 $\mathrm{dB}$ bandwidth is $380 \mathrm{MHz}$, which is somewhat less than needed, but we hope that implementation of output filter circuit would give $500 \mathrm{MHz}$, or $0.5 \%$ from carrying frequency.

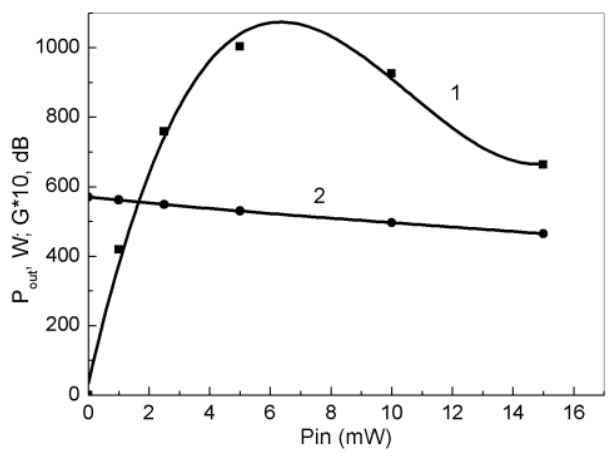

Fig. 4. Output power (1) and amplification factor (2) vs input power

The project confirmed the possibility of design and manufacturing 3-millimeter wavelength power klystron, using existing technological equipment.

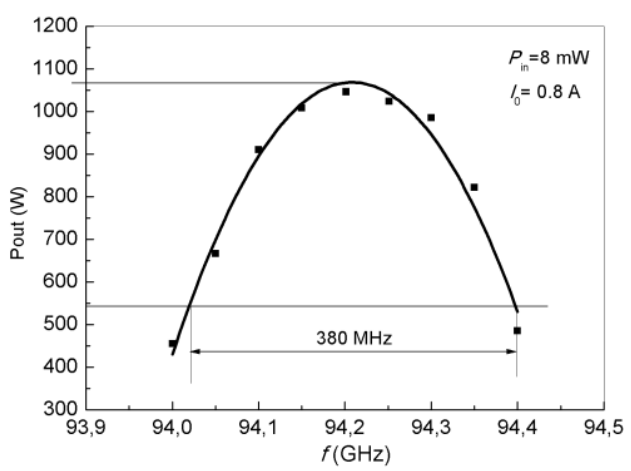

Fig. 5. Output power vs frequency

\section{References}

1. http://citeseerx.ist.psu.edu/viewdoc/download?doi=1 0.1.1.734.2121\&rep=rep1\&type $=$ pdf .

2. Cariotakis, G. High power klystrons: theory and practice at the Stanford Linear Accelerator Center // SLACPUB 10620. 2005. 138 pp.

3. Grigoriev A. D., Salimov R.V., Tikhonov R.I. Cellular handsets antenna modeling by vector finite element method // Journal of communication technology and electronics. 2012, V.57, No 3, pp 261-270. 